\title{
Ser sujeto hablante en el aula de Lengua Materna
}

\author{
Being a speaking subject in the mother \\ language classroom
}

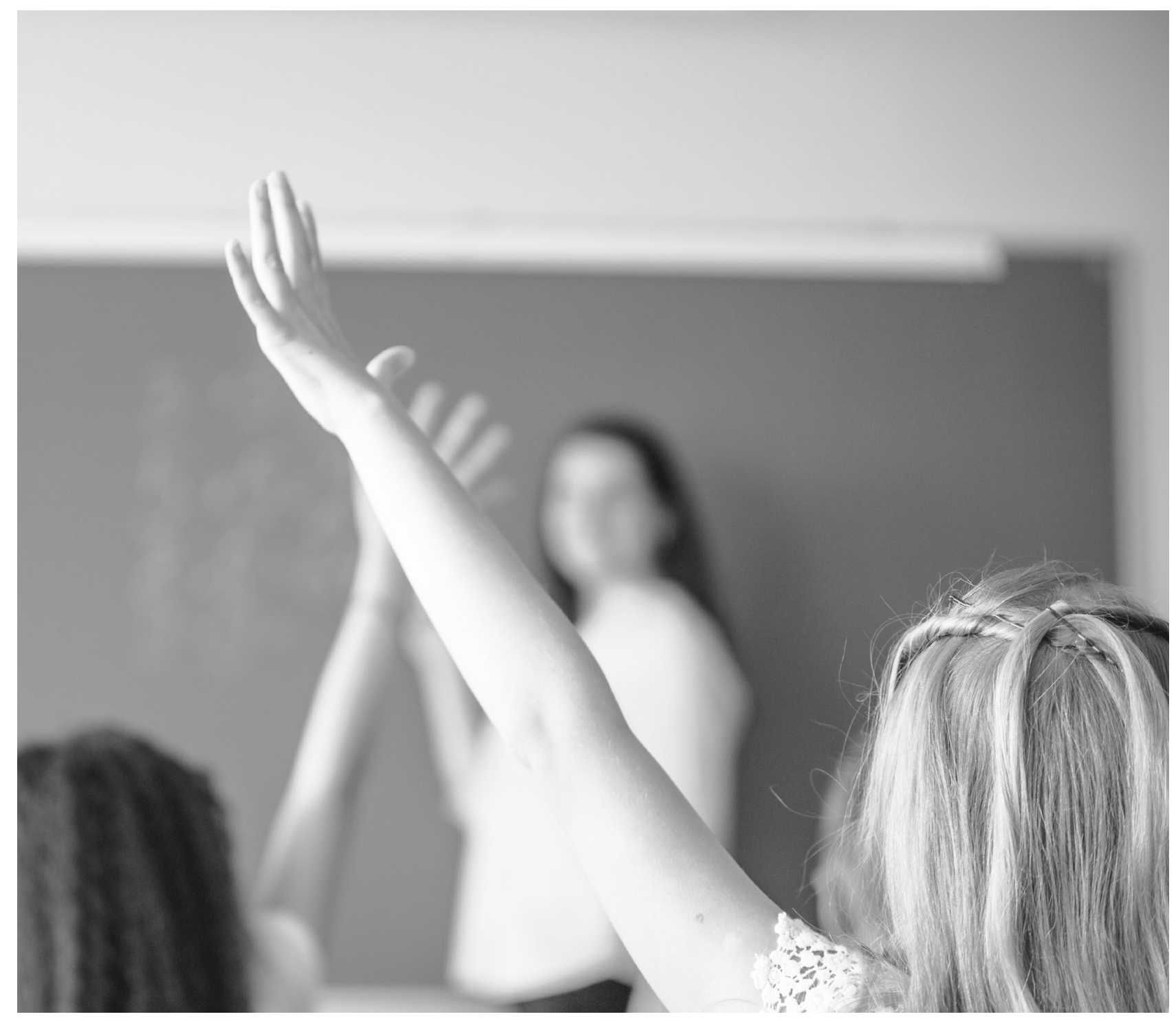




\title{
Ser sujeto hablante en el aula de Lengua Materna ${ }^{1}$ Being a speaking subject in the mother language classroom
}

\author{
Jhon Alexánder Monsalve Flórez²
}

Artículo recibido en marzo 30 de 2020; artículo aceptado en abril 24 de 2020

Este artículo puede compartirse bajo la Licencia Creative Commons Atribución-NoComercial-Compartirlgual 4.0 Internacional y se referencia usando el siguiente formato: Monsalve, J. A. (2020). Ser sujeto hablante en el aula de Lengua Materna. I+D Revista de Investigaciones, 15 (2), 28-37. DOI: https://doi.org/10.33304/revinv.v15n2-2020003

\begin{abstract}
Resumen
El presente artículo tiene como objetivo presentar orientaciones generales para la integración de la competencia ser sujeto hablante en el aula de Lengua Materna. Ante el uso lingüístico descontextualizado de los estudiantes del actual colegio Villas de San Juan, en Girón, Santander, se procede, desde el año 2016, a realizar una investigación cualitativa, bajo un paradigma sociocrítico, con el fin de que los estudiantes alcancen la competencia ser sujeto hablante, es decir, que adecúen sus intervenciones comunicativas en dependencia del contexto. Para ello, se parte de concepciones teóricas sistematizadas por Eugenio Coseriu en 1967, referentes a los entornos en los cuales se instauran las prácticas de habla y, además, de estudios realizados alrededor de la sociolingüística y la educación. Con base en esta descripción teórico-metodológica, se proponen algunas estrategias que, dentro de una secuencia didáctica, potenciarían esta competencia lingüístico-social en los estudiantes de bachillerato de la institución educativa.
\end{abstract}

Palabras clave: Actualización lingüística, educación, entorno lingüístico, ser sujeto hablante, sociolingüística.

\begin{abstract}
The objective of this article is to present general guidelines for the integration of the competence of being a speaking subject in the Mother Language classroom. Given the decontextualized linguistic use of the students of Villas de San Juan school, in Girón, Santander, a qualitative investigation has been carried out since 2016, under a sociocritical paradigm, in order for students to reach proficiency in being a speaking subject, that is, that they adapt their communicative interventions depending on the context. To do this, we turn to theoretical conceptions systematized by Eugenio Coseriu in 1967, referring to the environments in which speech practices are installed and, in addition, to studies carried out around sociolinguistics and education. Based on this theoretical-methodological description, some strategies are proposed that, within a didactic sequence, would enhance this linguistic-social competence in the high school students of this educational institution.

${ }^{1}$ Artículo de reflexión, con enfoque cualitativo, resultado de un proyecto de investigación culminado "Desarrollo de la competencia ser sujeto hablante en estudiantes de Educación básica y media de un colegio público de Girón, Santander", perteneciente al área de ciencias sociales, subárea educación y prácticas pedagógicas, desarrollado en el Grupo de Investigación Cultura y Narración en Colombia (Cuynaco) de la Universidad Industrial de Santander. Dirección: Carrera 27 calle 9. PBX: (057) 6344000. Fecha de inicio: julio de 2016. Fecha de terminación: noviembre de 2018. ${ }^{2}$ Magíster en Semiótica y Licenciado en Español y Literatura de la Universidad Industrial de Santander. Docente de carrera del colegio Villas de San Juan, en Girón. Profesor cátedra asociado de la Escuela de Educación de la Universidad Industrial de Santander. Vinculado al Grupo de Investigación Cultura y Narración en Colombia (Santander, Colombia). Dirección: Carrera 27 calle 9. PBX: (057) 6344000. ORCID ID: https://orcid.org/0000-00019853-9079. Correo electrónico: monsalve-jhon@hotmail.com.
\end{abstract}


Keywords: Linguistic actualization, education, linguistic environment, being a speaking subject, sociolinguistics.

\section{Introducción}

El presente artículo de reflexión se enmarca en la línea "Prácticas pedagógicas" del grupo de investigación Cultura y Narración en Colombia (Cuynaco), de la Universidad Industrial de Santander. Surge de una investigación que inicia en el año 2016, con el reconocimiento de prácticas lingüísticas estudiantiles en el aula de sexto grado de un colegio público de Girón, Santander. De manera general, el problema consiste en que los estudiantes, según la técnica de observación utilizada en el momento (Postic y Ketele, 2000), no adecúan de manera oportuna los usos de la lengua en dependencia de los contextos comunicativos. La investigación, desde sus comienzos, se enmarca en un paradigma sociocrítico de la ciencia, específicamente en el enfoque investigación-acción (McKernan, 1999; Torres, 1997); entre otros aspectos, se plantea como objetivo principal el desarrollo de la competencia ser sujeto hablante en los estudiantes de la Sede F del colegio Luis Carlos Galán Sarmiento, que, a partir del año 2019, es la sede principal del colegio Villas de San Juan.

Respecto a la competencia fundamental de la pesquisa, se propone el concepto ser sujeto hablante con el fin de dar cuenta de las habilidades sociocomunicativas pertinentes de los educandos en el momento de intervenir lingüísticamente. En una primera aproximación de este concepto (Monsalve, 2017), se describe una experiencia didáctica que tiene como objetivo el desarrollo de la competencia mencionada en el aula de clase de sexto grado. Un complemento de esta propuesta (Monsalve, 2018) tiene como fin presentar una metodología para el reconocimiento de la necesidad de potenciar la competencia ser sujeto hablante en los diferentes niveles de la educación. Esos trabajos dan pie para proponer ahora, en este artículo, unos fundamentos que permitan la integración adecuada de esta competencia en las metas que se estipulen para el área de Lengua Materna. Se parte del hecho de que los estudiantes, caracterizados por diversos usos de lengua propios de las comunidades a las que pertenecen, deben diferenciar los entornos, en términos de Coseriu (1967), para adecuar el uso que hacen de la lengua a las diversas circunstancias en las que se presentan las intervenciones discursivas.

Los referentes teóricos y metodológicos que sustentan la pesquisa surgen de estudios sociolingüísticos asociados con temas de educación; es decir, la investigación se basa en una sociolingüística aplicada de la lengua española: una suerte de integración entre la teoría de los entornos de Coseriu (1967) y la orientación formativa del uso de la lengua en el aula propuesta por Areiza et al. (2012); Giraldo (2016); Hernández (2001); y Stubbs (1984). En el desarrollo del presente artículo, se describe parte de los fundamentos teórico-metodológicos más trascendentales de estos investigadores, mientras se contraargumentan algunas de sus posturas y se problematiza sobre la norma, en sentido coseriano, que debe predominar en el aula de Lengua Materna.

La competencia ser sujeto hablante no puede potenciarse si se considera que, en todos los contextos, los estudiantes deben hacer uso de una norma estándar o, incluso, académica. El comportamiento profesoral frente a la competencia ser sujeto hablante implica una actitud comprensiva sobre las actualizaciones lingüísticas de sus estudiantes, tanto dentro como fuera del aula. Estas actitudes se logran no con la prescripción lingüística al estilo de la mejor gramática normativa, ni con una flexible disposición ante los hechos lingüísticos de los estudiantes en comunidades no académicas, tal y como lo proponen, en cierto momento, Areiza et al. (2012). La competencia ser sujeto hablante exige una mirada éticolingüística del habla, es decir, una actitud humana que permita la comprensión de las diferentes prácticas de habla como actividades propias del devenir lingüístico. No se trata, entonces, de prescribir u olvidar las normas académicas; el abordaje de la competencia ser sujeto hablante en el aula consiste en propender hacia un uso contextualizado y, por tanto, pertinente de la lengua.

Con el desarrollo de lo anterior, se presentan los fundamentos para integrar la competencia ser sujeto hablante en el aula de Lengua Materna. Se propone, por último, que la integración parta de secuencias didácticas que tengan presente alguna o varias de las siguientes actividades (entre otras que considere el docente): 1) el reconocimiento de las comunidades lingüísticas a las que pertenecen los educandos; 2) la relación entre literatura y diversidad lingüística y la reflexión en torno a cómo se habla en dependencia del contexto en el que los sujetos intervienen comunicativamente; 3 ) el uso de la lengua en las redes sociales y en el aula, y 4) el análisis por parte de los estudiantes y del profesor sobre cómo actúan lingüísticamente los compañeros de clase en los diversos entornos institucionales (recreo, aula, conversaciones mientras llega el docente, etc.) y en prácticas comunicativas ajenas a la escuela.

\section{Metodología}

El paradigma que rige la investigación en general es el sociocrítico, particularmente desde el enfoque participativo de la investigación-acción (McKernan, 1999; Torres, 1997). Para comprender el método aquí propuesto, es necesario que el lector sitúe los objetivos 
del presente trabajo como consecuentes de dos publicaciones anteriores (Monsalve, 2017, 2018). De este modo, aunque el método descrito en las próximas páginas sea más de orden teórico con fines propositivos, no necesariamente se aleja del paradigma sociocrítico, pues, en las últimas páginas, se proponen caminos para la intervención didáctica real en el aula.

El método utilizado para proponer lineamientos de integración de la competencia ser sujeto hablante en el aula de Lengua Castellana es complementario: por un lado, la teoría metodológica de la lingüística; por el otro, la teoría metodológica de la educación en Lengua Materna. La primera se sustenta en gran parte por Coseriu (1967), y la segunda, por diversos teóricos que han sabido asociar la lingüística con la didáctica de la lengua.

\section{Primera parte del método: Reconocimiento de los entornos desde la teoría de Eugenio Coseriu}

Coseriu (1967) describe cuatro tipos de entornos lingüísticos en los que participan los sujetos hablantes. Uno de ellos es la situación, comprendida como "el «espacio-tiempo» del discurso, en cuanto creado por el discurso mismo y ordenado con respecto a un sujeto" (p. 310). La situación es la que permite reconocer el destinador y el destinatario del proceso de comunicación, ubicado dentro de un ahora y un espacio actual. La situación no se centra en el contenido del mensaje expresado, sino en las características del discurso en cuanto este se produce. El entorno situacional no se entiende desde el pasado, sino desde el presente, desde lo que se enuncia por parte de los que intervienen en la comunicación. Si un sujeto hablante expresa "Yo te dije que te esperaría a mediodía en este lugar y no en la casa. ¿Por qué llegaste ahí?", plantea una situación que no debe percibirse desde el contenido del mensaje, sino desde el reconocimiento de quién y a quién habla, en qué espacio y en qué momento. La situación del enunciado anterior se determina a partir de un sujeto que habla en primera persona, se dirige a una segunda persona, cuando ha pasado la hora del encuentro (mediodía) y se encuentra en "este lugar", y no en la casa. La situación muestra el quién, a quién, el dónde y el cuándo del discurso que se profiere, y permite la comprensión deíctica de estos elementos a partir de los conocimientos discursivos de los participantes del proceso comunicativo. En el análisis situacional, no interesa de qué se habla, sino quién lo enuncia, a quién, dónde y cuándo.

El ser sujeto hablante se configura como tal dentro de este entorno, cuando reconoce las relaciones deícticas que se presentan en los enunciados y participa de manera pertinente ante el destinatario, lugar o momento en que se halla. En el contexto santandereano, quien es competente en el ser sujeto hablante se caracteriza por evitar tutear a personas cuya jerarquía social es notoria. No es habitual tratar de "tú" al jefe de una empresa cuando se tramita una carta administrativa dirigida a él. Además, el reconocimiento del tiempo y el espacio en que se desarrolla el acto comunicativo aporta a la intervención adecuada del sujeto hablante, es decir, brinda los fundamentos para saber qué decir y cómo decirlo. Si la comunicación se desarrolla en un ahora que sea el viernes de la Semana Mayor, en un espacio que sea la iglesia y cuyos interlocutores profesen la religión católica, lo que dicen y cómo lo dicen puede ser diferente a cuando participan en un ahora que sea una noche de cumpleaños y un espacio que sea un bar.

Otro entorno expuesto por Coseriu (1967) es la región, entendida como "el espacio dentro de cuyos límites un signo funciona en determinados sistemas de significación. Tal espacio está admitido, en un sentido, por la tradición lingüística $y$, en otro sentido, por la experiencia acerca de las realidades significadas" (p. 311). En la región interesa el reconocimiento del espacio que hace posible una actualización lingüística particular dentro de una situación. Puede estar caracterizada por la tradición lingüística, es decir, por las formas lingüísticas que, en cierto espacio geográfico, se han venido utilizando. También puede determinarse por experiencias en realidades significadas, en cuanto a cómo, según algunos subespacios, configuran otras maneras de abordar la lengua tras fines específicos. Por esta generalidad en la definición del concepto de región, Coseriu opta por proponer tres tipos de región en los procesos en los que intervienen sujetos hablantes. Uno de ellos es la zona, entendida como la delimitación lingüística que normalmente permite el reconocimiento de isoglosas; en palabras del lingüista: "La zona es la región en la que se conoce y se emplea corrientemente un signo; sus límites dependen de la tradición lingüística y suelen coincidir con otros límites, también lingüísticos" (p. 311). Según esto, una zona en Colombia sería lo que Montes (1982) denomina zona pacífica, en donde el uso de la lengua se da de manera particular -hasta el punto de diferenciarse de la zona caribeña-, gracias a unas tradiciones lingüísticas que han configurado el habla de los sujetos que en ella participan. El concepto de zona, como se evidencia, es más geográfico y, por tanto, determinante de la manera como se expresan los sujetos hablantes.

Otra categoría propuesta por Coseriu (1967), dentro del entorno regional, es el ámbito, entendido como "la región en la que el objeto se conoce como elemento del horizonte vital de los hablantes o de un dominio orgánico de la experiencia o de la cultura, y sus límites no son lingüísticos" (p. 311). El ámbito es el lugar en el que se configuran otras formas lingüísticas para abordar los mismos $u$ otros hechos de la experiencia humana cotidiana a partir de otros significantes. El ámbito 
permite reconocer el uso de lengua específico; Coseriu (1967) lo asocia directamente con voces técnicas. Dentro de las zonas, puede haber algunas variedades léxicosemánticas que se reconocen debido a la tradición lingüística de las comunidades que hacen parte de ellas. No es de extrañar que en un poema como "Carta provinciana", de Castro (1973), se hallen palabras como chumado para hacer referencia a borracho -al menos en la situación lingüística en la que se presenta al yo poético-. Y no es extraño porque la tradición lingüística de esa zona ha permitido el uso de esta palabra de generación en generación por parte de los campesinos de Nariño, que no es usada, por ejemplo, por los campesinos de Santander. En el ámbito, por el contrario, las variaciones no se dan a causa de una tradición lingüística popular, sino por una terminología propia de especialidades en el ámbito de la ciencia. Retomando un ejemplo de Coseriu, la expresión "Tiene dolor de cabeza" es muy común dentro de ciertas zonas, pero no lo es tanto en ciertos ámbitos, tales como la medicina, en donde podría ser más usual el enunciado "Sufre de cefalalgia". La densidad terminológica o el metalenguaje de cada área determinan un uso especial de la lengua (propios del ámbito) que se construye en espacios diferentes al de la cotidianidad y al de los límites geográicos (propios de la zona).

Otra área donde también se configuran prácticas lingüísticas diferentes es el ambiente, comprendido como "una región establecida social o culturalmente. La familia, la escuela, las comunidades profesionales, las castas, etcétera, en cuanto poseen modos de hablar que les son peculiares, son 'ambientes"' (pp. 311-312). En el ambiente influyen las relaciones sociales que conforman comunidades lingüísticas que pueden estar englobadas dentro de zonas o ámbitos lingüísticos. Por ejemplo, el uso de lengua que se da en familia puede estar asociado, en parte, con la tradición lingüística de la comunidad a la que pertenezcan sus participantes. Sin embargo, este grupo social determina formas de hablar diferentes a otras que pertenecen a la misma zona; por ejemplo, las formas de hablar de la familia pueden ser divergentes a las formas de hablar entre amigos, aunque pertenezcan al mismo entorno zonal. Esto se evidencia, por ejemplo, en algunos discursos de los estudiantes que varían según se comuniquen con sus amigos o con sus padres de familia. Bien puede hallarse el caso de un adolescente que, con sus padres, habla sin hacer uso de palabras consideradas como groseras, pero que, en el ambiente entre amigos, se dirige hacia ellos con disfemismos (Lázaro Carreter, 1998); por ejemplo, un joven de la comunidad del ñerismo bumangués puede dirigirse a otro utilizando el adjetivo "pichurria" no para insultarlo, sino para demostrar lazos de proxemia entre los dos; sin embargo, frente a sus padres de familia este mismo sujeto no utiliza tales expresiones porque reconoce el ambiente tan diverso en el que se halla.
Si se retoma este último ejemplo, podría hacerse alusión a un uso pertinente de la lengua por parte del adolescente, que reconoce el ambiente en el que sucede su discurso para actuar adecuadamente en él. Podría decirse que el interlocutor, en este caso, es competente, sabe ser sujeto hablante. No interesa, y no está de más decirlo, que el sujeto hablante haya utilizado una expresión que, desde un acto prescriptivista y hasta moralista de la lengua, puede ser considerada grosera. La competencia del ser sujeto hablante parte de una perspectiva descriptiva y funcionalista cuyo objetivo no es decir qué está bien o mal cuando se habla, sino indicar qué y cómo debe hablar un sujeto según la situación, la región, el contexto o el universo del discurso en que se encuentre.

En el caso del entorno regional, específicamente desde la zona y el ámbito, el ser sujeto hablante se potencia según el reconocimiento de su tradición lingüística y de las voces técnicas que caracterizan ciertos discursos. Es normal que un sujeto nacido en Santander utilice, en situaciones cotidianas, el vocativo "mano", propio de esta zona, y es poco habitual que utilice, en su lugar, el vocativo "toche", más común en Norte de Santander, específicamente en Cúcuta. Un santandereano sabe ser sujeto hablante cuando utiliza elementos característicos de su zona, sin considerarlos retrógrados o poco cultos. También hace un uso apropiado de la lengua en ámbitos en los que participe y que determinen otros usos lingüísticos. Verbigracia, la palabra isotopía, que es propia de las ciencias del lenguaje (Greimas et al., 1990), determina una recurrencia semántica en los discursos, y dentro de este ámbito es apropiada. Considerar que, en una conversación entre amigos, es preferible esta palabra a recurrencia, reiteración o redundancia es extrapolar las prácticas lingüísticas de una ciencia a entornos en los que tal utilización no es pertinente.

Uno de los entornos más reconocidos de la teoría coseriana es el contexto, entendido como "toda la realidad que rodea un signo, un acto verbal o un discurso, como presencia física, como saber de los interlocutores y como actividad" (p. 313). El contexto se comprende desde tres categorías; la primera de ellas es la verbal. Independientemente del texto que surja en el proceso de comunicación, los hablantes hablan sobre algo. El tema del discurso configura su contexto verbal. Dependiendo de la temática, el uso de la lengua puede variar. Por ejemplo, si se habla de política, es muy común que términos como congreso de la república, constitución política, leyes, decretos, etc., sean utilizados por los interlocutores, lo que no sucedería si el discurso gira en torno a la última fiesta o rumba entre amigos.

Aunque Coseriu (1967) propone para la categoría de contexto extraverbal seis subtemas, para el presente trabajo interesa, sobre todo, el contexto práctico $u$ ocasional, debido a la relación directa que existe entre 
este y el uso pertinente de la lengua de un sujeto en una ocasión particular. El lingüista explica la importancia de esta segunda categoría en casos como:

[...] el hablar con un anciano o con un niño, con un amigo o con un enemigo, para pedir un favor o para exigir un derecho; el acontecer el discurso en la calle o en una reunión familiar, en una clase o en el mercado, de día o de noche, en invierno o en verano, etc. (p. 316)

Este contexto determina formas de hablar que varían en dependencia de la ocasión, del interlocutor, del lugar o el momento en que se lleva a cabo el discurso. Es innegable que este contexto ocasional se relaciona estrechamente con el situacional.

La competencia del ser sujeto hablante se potencia, en este contexto, cuando el sujeto reconoce los espacios, las personas y los momentos para intervenir oportunamente en el género textual que habla o escribe. No sería competente, por ejemplo, un estudiante que, en una sustentación académica, inicie su discurso con un vocativo como "ñeritos" (bastante usado por los hablantes pertenecientes a la comunidad del ñerismo en el área metropolitana de Bucaramanga), pertinente en otra ocasión, fuera del aula y entre amigos, e inapropiado en la escuela, en una exposición entre compañeros de clase y profesores.

La tercera categoría es más simple y parece intervenir en menor proporción en la definición de ser sujeto hablante. Este contexto se denomina idiomático y hace alusión a que los sujetos hablantes en el mundo se comunican bajo las normas de sistemas y subsistemas que caracterizan la variación constante de la lengua que hablan. Entonces, ¿lo único que habría que decir aquí es que, en Colombia, por ejemplo, un sujeto sabe ser sujeto hablante cuando habla español? Lo que parece ser algo más que habitual se enfrenta a retos con los préstamos lingüísticos que se hacen de otras lenguas. Aquí sí sería apropiado preguntarse por la pertinencia de los anglicismos o galicismos en diferentes situaciones, regiones o contextos para determinar si el que habla sabe ser sujeto hablante.

El universo del discurso, que es el último entorno propuesto por Coseriu, se entiende como "el sistema universal al que pertenece un discurso (o un enunciado) y que determina su validez y su sentido" (p. 318). Esta categoría debe percibirse de manera general y como configuradora englobante de los demás entornos. Verbigracia, el uso de terminología propia de las matemáticas da cuenta de un universo discursivo diferente al de la cotidianidad entre amigos. Los ámbitos configuran, así las cosas, actualizaciones de diferentes universos del discurso. Estos no son más que sistemas configurados por normas implícitas en comunidades, ambientes, ámbitos, zonas, situaciones o contextos que permiten reconocer el uso adecuado que merece la lengua. Cuando se abordan las categorías de entornos lingüísticos expuestas en páginas precedentes, se hace siempre en función de un universo del discurso en el que se instaura la práctica lingüística. A partir del enunciado "Se salió por la tangente", es importante reconocer un universo discursivo que explica al otro. Esta, que es una expresión coloquial, propia de un universo del discurso cotidiano y general, se entiende gracias a la conceptualización de tangente desde la trigonometría, otro universo de discurso. Estos universos se actualizan, entonces, en ámbitos, situaciones o contextos, cuando de ciencia, tecnología o simple cotidianidad se trata.

Como se evidencia en esta teoría coseriana, el estudio de los entornos configura la pertinencia de la intervención lingüística de los sujetos hablantes. La intención de las páginas siguientes no es más que asociar esta teoría con la práctica habitual del aula de clase. Para ello, se tendrán en cuenta didactas de la lengua y lingüistas que han relacionado los estudios de la ciencia de la lengua con los haceres didácticos.

\section{Complemento del método con teoría educativa: uso de la lengua dentro y fuera del aula}

¿Es necesario diferenciar los usos que los estudiantes hacen de la lengua dentro y fuera del aula de clase? ¿Es importante orientar al estudiante en el uso contextualizado de sus prácticas lingüísticas? ¿Se hace significativa una actualización académica por parte del docente en un aula cuyos estudiantes no estén familiarizados con tales prácticas? ¿No sería mejor que el docente adecuara su discurso al registro y contexto habitual de los estudiantes? ¿Es necesario diferenciar unos usos con respecto a otros, si la comunicación, independientemente de cómo se realice, cumple su propósito ilocutivo?

Las preguntas anteriores hacen parte de los problemas que identifica el investigador de la propuesta ser sujeto hablante, en las aulas de bachillerato del colegio Luis Carlos Galán Sarmiento de Girón, Santander (hoy llamado colegio Villas de San Juan). Además, surgen de diferentes teóricos que han abordado el tema, pero con ciertas aristas. Areiza et al. (2012), por ejemplo, invitan a pensar en que "el sistema educativo debe abrirle alternativas comunicativas al estudiante para abordar problemas académicos, utilizando su propia variante lingüística para expresar su mundo conceptual y desempeñarse en su ejercicio cognitivo y comunicativo" (p. 182). Esta propuesta debe ser tomada con pinzas y no desligada de la precisión que hacen los autores en párrafos posteriores: "[...] las formas del habla no estándar son instrumentos válidos con los cuales los niños pueden 
abordar, por lo menos en los niveles más elementales, los contenidos académicos de la educación formal" ( $p$. 182). Pero, aun así, ante esto es importante cuestionar si la inclusión de formas lingüísticas propias de los estudiantes, que pueden llegar a ser tan heterogéneas dentro de un aula de clase, es una estrategia apropiada para que los niveles de pérdidas de asignaturas o de deserción disminuyan.

La escuela es un entorno diferente al de la calle o al de la familia. La escuela determina unas formas lingüísticas particulares, relacionadas con diversos ámbitos y universos del discurso. Si bien Areiza et al. (2012) aclaran que la inclusión en la escuela de variantes propias de los ámbitos en los que están habituados los educandos se podría hacer, al menos, en los primeros años de formación, también es cuestionable que dentro de un entorno académico se integren prácticas lingüísticas de un ámbito cuyas voces sean usuales y no técnicas. ¿Cómo explicar, por ejemplo, qué es un múltiplo o un divisor con la utilización lingüística de los niños que pertenecen a comunidades del ñerismo del área metropolitana de Bucaramanga? ¿Cómo hacerlo cuando los grupos son tan heterogéneos, es decir, cuando existe más de una variante dentro del aula? La intención pedagógica es aceptable, pero la realización es bastante compleja. Es necesario que los estudiantes de primeros niveles (y de los que siguen), como sujetos hablantes, se adecúen a nuevos contextos de interacción comunicativa, mediante estrategias de uso discursivo por parte de los profesores. Esto indica que los docentes no orienten sus procesos haciendo uso de las prácticas lingüísticas de los estudiantes, sino en función de la edad de ellos y de su nivel cognitivo.

Dentro de un ámbito académico perteneciente a un universo del discurso científico, no es pertinente el uso de actualizaciones lingüísticas propias del ámbito familiar, donde el niño, normalmente, no escucha sobre ciencia, sino sobre vida, juegos, problemas, viajes, paseos, etc. Estos ámbitos tan diversos merecen una práctica diferente en el uso de la lengua. Además, la situación cambia: en familia, el niño puede dirigirse a los padres con el fin de solicitar dinero para el recreo, mientras al profesor se dirige para resolver alguna duda sobre matemática o literatura. Esto no quiere decir que los ámbitos no se integren o extrapolen. Bien puede

\footnotetext{
${ }^{3}$ Hernández (2001), quien parte del concepto de norma lingüística desarrollado por Coseriu, propone la enseñanza de la lengua estándar en la escuela. Considera importante tener en cuenta todas las variedades lingüísticas, sin juicios de valor en torno a ellas, pero también afirma que, independientemente de la norma lingüística a la que pertenezca el estudiante (esto es, sin tener en cuenta las prácticas lingüísticas propias del estudiante en diversos contextos), es necesario que entienda y se comunique gracias a un elemento que sea común a todas las variedades: "Existen, sí, pluralidad de normas, pero sobre todas, como su máximo común denominador por una parte, y su mínimo común múltiplo por otra, está la norma estándar, dúctil,
}

presentarse el caso de un niño que pida dinero a la profesora para el descanso, porque tiene sensación de hambre, o que solicite ayuda a sus padres de familia para realizar una tarea. Lo cierto es que estas situaciones, también comunes, se emparentan con el ámbito familiar y académico, respectivamente, pero bien se reconoce que no son prioritarias ni en un entorno ni en el otro. Es más, cuando el padre de familia explica una tarea a su hijo, es posible que lo haga con vocativos diferentes, con palabras que nacen de impaciencias y con ausencia de terminología adecuada para tal fin, o con desconocimiento de procesos similares con los que se logran los mismos objetivos. Algo similar ocurre con la profesora que le explica al niño que no tiene dinero, que le queda solo lo del bus o que olvidó la billetera. Estas explicaciones no son académicas, sino, más bien, familiares o cotidianas a las que la profesora debe acudir para entablar una conversación ya no académica, sino propia de otro ámbito ajeno al escolar. Pero incluso en este último caso, basta para comunicarse con el niño un uso estandarizado ${ }^{3}$ de la lengua y no un uso particular (por ejemplo, el ñerismo) que devenga de las situaciones familiares o barriales a las que está habituado el estudiante.

Todo lo anterior apunta a una actualización adecuada de la lengua dentro y fuera del contexto académico. No se pretende con esta propuesta prescribir formas de hablar, ni tampoco condenar a quienes utilicen cierto léxico que no ha sido aprobado por la Real Academia de la Lengua Española. Como se aclaró al principio de este trabajo, el objetivo no es otro que reconocer las variantes de una lengua y los contextos en los que se utilizan para actuar pertinentemente en torno a ellas. Así las cosas, no se trata de que el niño deje de hablar como habla en familia, sino, más bien, de que tenga conciencia de que en la escuela se habla de otra manera, que exige, por ejemplo, la adecuación de la lengua, tal como lo afirma Giraldo (2016), "de suerte que reflexionen sobre lo expresado arbitrariamente y asuman, de acuerdo con los ámbitos formales e informales en los que interactúen, formas significantes adecuadas" (p. 74).

Si lo que se viene argumentando podría resultar evidente, ¿por qué razón los niños no se comportan de manera adecuada, en el ámbito lingüístico, dentro del aula de clase? ¿Por qué las exposiciones siguen plagadas de

flexible, que permite la interacción comunicativa de todo y en todo el mundo hispanohablante". Esta propuesta, aunque necesaria y muy bien argumentada, no es apropiada en la teorización del ser sujeto hablante, por cuanto no considera prioritarios los usos particulares de la lengua en contextos diversos (por ejemplo, ¿se puede hablar de lengua estándar en la enseñanza de la trigonometría? O sea, ¿se puede explicar una hipotenusa sin hacer alusión a este término?). Lo cierto es que, en el ejemplo propuesto, la profesora opta por una actualización estándar de la lengua pertinente en el momento y para el nivel cognitivo del niño, que también entiende, sin necesidad de que la docente trascienda a los usos lingüísticos del ámbito familiar o barrial. 
usos de la lengua anormales para el ámbito en el que se hallan? ¿Por qué los vocativos utilizados para referirse a sus compañeros son característicos de las relaciones que entablan entre amigos y no entre académicos? La razón, desde la postura del autor de este trabajo, se basa en que no son competentes en el ser sujeto hablante. Según los avances que se han realizado en torno a este tema (Monsalve, 2017, 2018), se concluye que los estudiantes no saben actualizar su práctica lingüística según el entorno en el que se comunican, debido a una construcción identitaria muy fuerte de su hacer lingüístico en ámbitos ajenos al escolar, y a una falta de orientación por parte del docente, que tiende más a cuestionar lo mal que hablan los estudiantes que a analizar las razones de tales usos en la escuela para trabajar en torno a ello. La siguiente apreciación de Stubbs (1984) permite una reflexión sociolingüística sobre este hecho:

[...] muchos profesores mantienen la creencia de que solo hay una lengua "óptima" para todos los propósitos, y que esta es la única lengua adecuada en la clase. Sin embargo, un momento de pensamiento o de observación convencería a cualquier profesor de que ellos mismos son los primeros en utilizar muchas variantes lingüísticas a lo largo del día, dependiente del propósito o contexto de la comunicación. Esto no es reprensible, ni implica una variabilidad camaleónica, sino que es un rasgo sociolingüístico básico de uso del lenguaje en todo el mundo. (p. 36)

Ningún sujeto hablante, ante diferentes ámbitos o contextos, habla exactamente igual. El estudiante debería considerar que la lengua varía según la circunstancia en la que se encuentre, y que, por tal motivo, no debe dejar de utilizar el léxico que caracteriza su intervención comunicativa en ambientes diferentes al escolar, pero tampoco debe utilizarlo en ámbitos como el académico. Así mismo, los docentes deberían considerar la variación lingüística como un rasgo propio de las lenguas, que es inabarcable e imprescribible en su totalidad y que brinda identidad a sus estudiantes en grupos diversos. El hecho de criticar esas expresiones lingüísticas puede ser contraproducente para la actitud del sujeto, pues al ser consideradas hablas sin cultura, "el niño prefiere abandonar la escuela antes que traicionar el sentimiento de lealtad que lo apega a su mundo cultural. Inconscientemente, se siente burlado" (Areiza et al., 2012). Pero no por ello, y atención al énfasis, el docente dejará de orientar el uso adecuado de la lengua

\footnotetext{
${ }^{4}$ Se insiste en que este ciclo escolar no es camisa de fuerza para implementar las actividades descritas. Bien pueden consultarse otras competencias en el documento del MEN (2006), donde se hallan propósitos similares. Por ejemplo, en décimo y undécimo grado: "Identifico, caracterizo y valoro diferentes grupos humanos teniendo en cuenta aspectos étnicos, lingüísticos, sociales y culturales, entre otros, del mundo contemporáneo" (p. 41); en octavo y noveno,
}

en los diferentes contextos.

Para Stubbs (1984), se juzga "precisamente inapropiado el empleo de coloquialismos y de formas dialectales regionales en una entrevista para solicitar empleo, de la misma forma que lo es el usar un lenguaje muy formal tomando una copa con unos amigos en una cafetería" (p. 37). Aquí se evidencia la adecuación, según el contexto en el que se desarrolla la práctica comunicativa. No se trata de negar los usos de lengua, sino de saber utilizarlos según la situación, el ámbito, el contexto o el ambiente.

De esta manera, se logra lo que para Hymes y Bernal (1996) es parte de la competencia comunicativa:

Tenemos entonces que explicar el hecho de que un niño normal adquiere el conocimiento de oraciones no únicamente en lo relativo a lo gramatical, sino también a lo apropiado. El niño adquiere la competencia relacionada con el hecho de cuándo sí y cuándo no hablar, y también sobre qué hacerlo, con quién, dónde y en qué forma. (p. 22)

\section{Resultados}

\section{Orientaciones generales para la integración de la competencia ser sujeto hablante en el aula de Lengua Materna}

De antemano, se hace claridad sobre el hecho de que la competencia ser sujeto hablante se potencia a lo largo de los años escolares; es decir, no se comprende como un estado, sino como un proceso. De este modo, aunque las orientaciones se lleven a cabo a lo largo de la primaria o del bachillerato, se propone a continuación un camino, posiblemente entre tantos, para integrar esta competencia en el aula de Lengua Materna, aunque bien se sabe que no es solo en esta área donde los estudiantes intervienen comunicativamente. Elegir el año escolar en donde sea pertinente una secuencia didáctica depende, en gran medida, de las necesidades que al respecto note el docente dentro y fuera del aula (en exposiciones, en el recreo, en conversaciones informales no académicas, etc.). Se ha considerado conveniente -pero no es camisa de fuerza- que la reflexión enfática sobre las diferentes formas de hablar se realice entre sexto y séptimo grado, debido a la organización propuesta por el Ministerio de Educación Nacional [MEN] (2006) en los Estándares Básicos de Competencias ${ }^{4}$. Según esta orientación

"Entiendo la lengua como uno de los sistemas simbólicos producto del lenguaje y la caracterizo en sus aspectos convencionales y arbitrarios" (p. 39); en cuarto y quinto, "Identifico los elementos constitutivos de la comunicación: interlocutores, código, canal, mensaje y contextos" (p. 35), y en primero, segundo y tercero, "Identifico en situaciones comunicativas reales los roles de quien produce y de quien interpreta un texto" (p. 33). 
pedagógica, es importante potenciar la siguiente competencia de orden cognitivo: "Identifico en situaciones comunicativas auténticas algunas variantes lingüísticas de mi entorno, generadas por ubicación geográfica, diferencia social o generacional, profesión, oficio, entre otras" (MEN, 2006). Este reconocimiento permite una reflexión metacognitiva sobre cómo se habla en diferentes ámbitos y contextos o, en otras palabras, en dependencia de aspectos diastráticos, diatópicos o diafásicos. A partir de ello, se proponen a continuación ciertas actividades que, utilizadas en una secuencia didáctica, pueden contribuir al desarrollo de la competencia ser sujeto hablante.

\section{El reconocimiento de las comunidades lingüísticas a las que pertenecen los educandos}

En primer lugar, el sujeto hablante debe reconocerse identitariamente: saber quién es, a qué comunidades de habla pertenece, cuáles son las características de su discurso, por qué habla diferente a otros sujetos de su misma edad, por qué en su departamento utilizan palabras y acentos diferentes a otros lugares de Colombia o de Hispanoamérica, cómo habla en casa, cómo lo hace en el recreo y cómo actualiza su lengua frente a los compañeros en una actividad académica. Para ello, puede realizar una descripción de su hacer lingüístico, a partir de las categorías mencionadas que determinan su identidad. Además, puede entrevistar a sujetos hablantes de su propia comunidad o familia haciendo uso de las mismas categorías. De este ejercicio, surgen debates en clase o exposiciones sobre cómo y por qué los estudiantes pertenecientes a la institución hablan de cierta manera y no de otra.

\section{La relación entre literatura y diversidad lingüística}

Álvarez (1987) presenta una nueva perspectiva sobre la clásica pureza de los escritos literarios. Después de poner como ejemplo a Rulfo (1981) y a García Márquez (1975), concluye que las expresiones literarias, de un tiempo para acá, han tenido en cuenta las variedades lingüísticas de las zonas en las que se contextualizan los textos narrativos y literarios. Sobre literatura y actualizaciones lingüísticas hay cuentos y novelas que pueden ser muy útiles: "La mujer", de Juan Boch, "El rifle", de Tomás Carrasquilla, cualquier novela de Fernando Vallejo, Rayuela, de Julio Cortázar (específicamente, los apartados en los que aparece el gíglico), "Carta provinciana", de José Félix Castro, "La muerte en calle", de José Félix Fuenmayor, etc. Los estudiantes leen los textos literarios y reflexionan sobre las variedades lingüísticas utilizadas por los personajes. En esta actividad, los estudiantes pueden responder preguntas como ¿por qué algunos actores literarios utilizan cierta actualización lingüística y otros no? Este interrogante permite la reflexión del tipo de variación que caracteriza el habla del personaje: diastrática, diafásica o diatópica (Coseriu, 1992). De aquí surge también la reflexión en torno a cómo se habla en dependencia del contexto en el que los sujetos intervienen comunicativamente.

\section{El uso de la lengua en las redes sociales y en el aula}

Los estudiantes comparan cómo se escribe un texto académico facilitado por el docente y cómo hablan ellos en Facebook o en WhatsApp. La asimilación permite una reflexión sobre el uso adecuado de la lengua en contextos diferentes. No se trata, en este caso, de afirmar que la manera como se utiliza la lengua en las redes sociales es correcta o incorrecta, sino, más bien, se analiza la impertinencia de usar palabras incompletas o representadas por grafemas con otras cargas semánticas (como "q" para indicar "que" o como " $x$ " para indicar "por") en el ámbito académico. Se da cuenta de que las situaciones, los contextos, los ámbitos y los ambientes configuran formas de hablar normales dentro de esos entornos.

\section{Evaluación por parte de los estudiantes sobre cómo actúan lingüísticamente los compañeros de clase en los diversos entornos institucionales (recreo, aula, conversaciones mientras llega el docente, etc.) y en prácticas comunicativas ajenas a la escuela}

Por último, se propone una auto- y coevaluación constante sobre cómo hablan los estudiantes en actividades de clase y en ámbitos ajenos al académico. Se recomienda la utilización de una bitácora para plasmar las memorias o conclusiones del día con respecto a la actividad. A partir de ello, puede plantearse un ejercicio de escritura de un comentario descriptivoargumentativo sobre cómo hablan los compañeros de clase tanto dentro como fuera del aula y en situaciones académicas y no académicas.

\section{Conclusiones}

A lo largo de este artículo, se ofrecen los fundamentos para integrar la competencia ser sujeto hablante en el aula de Lengua Materna. En primer lugar, se sitúa al docente en una perspectiva lingüística sobre el reconocimiento de los entornos y de la adecuación del sujeto hablante para actuar pertinentemente en su práctica comunicativa. En segunda instancia, se presenta un marco teóricometodológico en el que se relaciona la teoría lingüística con la didáctica de la lengua materna. El propósito de este apartado -además de entablar la necesidad de orientar al estudiantado en las maneras propicias de actuar lingüísticamente- era partir de autoridades en el tema para argumentar la pertinencia de desarrollar o potenciar la actitud de ser sujeto hablante. Por último, se otorgan algunas orientaciones para integrar esta competencia en el aula de Lengua Materna; entre otras, se 
tiene en cuenta el reconocimiento identitario del sujeto hablante, la relación entre literatura y sociolingüística y las diferencias entre la lengua en redes sociales y en la academia. El ser sujeto hablante se configura, teniendo en cuenta lo anterior, como una competencia necesaria no solo para actuar apropiadamente en los entornos comunicativos, sino también para comprender a los otros como interlocutores válidos, sin prejuicios sobre su actualización lingüística. Esta competencia se asocia muy notoriamente a un ser: ser mejor persona, ser mejor hablante, ser mejor humano.

\section{Agradecimientos}

El investigador agradece a la comunidad educativa del colegio Villas de San Juan, en Girón, Santander (antigua Sede F del colegio Luis Carlos Galán Sarmiento), por permitir el desarrollo de esta investigación a lo largo de los últimos 4 años. Así mismo, agradece el espacio del Grupo de Investigación Cultura y Narración en Colombia (Cuynaco) para este tipo de propuestas dentro de su línea "Prácticas pedagógicas".

\section{Referencias}

Álvarez, J. (1987). Didáctica de la lengua materna. Un enfoque desde la lingüística. Akal.

Areiza, R., Cisneros, M. y Tabares, L. (2012). Sociolingüística: enfoques pragmático y variacionista (Segunda ed). ECOE ediciones.

Castro, J. (1973). Antología de la poesía nariñense. Editorial publicitaria.

Coseriu, E. (1967). Teoría del lenguaje y lingüística general. Editorial Gredos.

Coseriu, E. (1992). Competencias lingüísticas: Elementos de la teoría del hablar. Editorial Gredos.

García Márquez, G. (1975). El otoño del patriarca. Círculo de lectores.

Giraldo, L. A. (2016). Forma y sentido de la ciencia del lenguaje. Fundamentos teóricos y prácticos del discurso lingüístico, pedagógico y juvenil (Primera ed). Universidad Tecnológica de Pereira.

Greimas, A., Campodónico, H., Courtés, J. y Ballón, E. (1990). Semiótica: diccionario razonado de la teoría del lenguaje. Gredos.

Hernández, C. (2001). ¿Qué norma enseñar? Actas Del Il Congreso Internacional de La Lengua Española. http://congresosdelalengua.es/valladolid/ponencias/ unidad_diversidad_del_espanol/1_la_norma_hispanica/ hernandez_c.htm

Hymes, D. H. y Bernal, J. G. (1996). Acerca de la competencia comunicativa. Forma y Función, (9), 13-37.

Lázaro Carreter, F. (1998). Diccionario de términos filológicos. Editorial Gredos.

McKernan, J. (1999). Investigación-acción y currículum: métodos y recursos para profesionales reflexivos. Ediciones Morata.
Ministerio de Educación Nacional. (2006). Estándares Básicos de Competencias en Lenguaje, Matemáticas, Ciencias y Ciudadanas (Ministerio de Educación Nacional, ed.). https://www.mineducacion.gov. co/1621/articles-116042_archivo_pdf.pdf

Monsalve, J. A. (2017). Sociolingüística en sexto grado: hacia una formación del ser-sujeto-hablante. Lingüística y Literatura, 38(72), 100-113. https://doi. org/10.17533/udea.lyl.n72a05

Monsalve, J. A. (2018). Fundamentos para la comprensión del ser sujeto hablante, desde el enfoque de la Lingüística del hablar. En L. F. Arévalo Viveros y Y. A. Nieto Lascarro (eds.), Encuentro con la lingüística, la semiótica y la enseñanza de lenguas (pp. 275-302). Universidad Industrial de Santander.

Montes, J. J. (1982). El español de Colombia: Propuesta de clasificación dialectal. Thesaurus: Boletín Del Instituto Caro y Cuervo, 37(1), 23-92.

Postic, M. y Ketele, J. M. (2000). Observar las situaciones educativas (3a edición). Narcea Ediciones.

Rulfo, J. (1981). Pedro Páramo. Editorial Bruguera.

Stubbs, M. (1984). Lenguaje y escuela. Editorial Cincel.

Torres, A. (1997). Enfoques cualitativos y participativos en investigación social. UNAD. 